\title{
Meta-Analysis as a Symptom: The Example of Flow Diverters
}

$\mathbf{T}$ he principle of a meta-analysis is to evaluate the effect of a treatment by combining the results of several studies. This method increases the statistical power to test certain hypotheses, especially when several randomized controlled trials are each too small to provide an answer or have provided contradictory conclusions.

Because we have very few randomized trials in interventional neuroradiology, our meta-analyses most often collect data of uncontrolled case series. A meta-analysis then makes sense when the target pathology is rare, with a limited number of cases reported in each publication. However, in this case, a single meta-analysis is sufficient, even if it may need to be updated sometimes.

The excessive use of multiple meta-analyses for the same medical problem is a symptom: the hope that the next one will provide better results than the previous one. Any neuroradiologist can consult PubMed and enter the term, object of his or her research, followed by "AND meta-analysis" and compare the number of responses obtained. Between February 2013 and January 2020, 19 meta-analyses, often redundant, have been published concerning the treatment of cerebral aneurysms by flow diverters. It is at this point that it would become possible to envisage a meta-analysis of meta-analyses!

I know that not all cerebral aneurysms are the same and that it may be necessary to distinguish those of the carotid from those of the vertebrobasilar circulation, to separately examine large and small aneurysms, those of the anterior communicating artery and those of the middle cerebral artery, those treatments for which one antiplatelet has been prescribed rather than another, and so forth. However, all these remarks also apply to coil treatment, and there have been only 2 published meta-analyses on that topic in the 27 years that they have been in clinical use. Why is that?

http://dx.doi.org/10.3174/ajnr.A6594
When a treatment has convinced a discipline of its efficacy and relative safety, meta-analyses are not necessary. There is no metaanalysis concerning usefulness of antibiotic treatment in pulmonary tuberculosis.

This overabundance of meta-analyses expresses the desire to convince physicians and patients of the benefits of this implant. Quantity makes quality. Most meta-analyses (as well as most case series) on flow diversion report a cumulative morbidity/mortality rate of $10 \%$ or so, as if this was the normal price to pay for "otherwise untreatable" aneurysms. The argument that flow diversion should fill a therapeutic void is not supported by the number of units used between 2 interventional neuroradiologist centers that treat roughly the same number of aneurysms per year. The regulatory status of implants allows them to be used in indications different from those for which they were initially presented to the health authorities (at least in Europe). Thus, large and giant aneurysms of the carotid siphon, the initial indication for flow diverters, are now only an excuse to extend their use in the treatment of small or even very small aneurysms in multiple other locations, and to "accept" the $10 \%$ complication rate without balancing this risk with the one of other management options, including observation. The apparent aim of multiplying metaanalyses may finally be to replace the unquestionable methodologic evaluation, ie, the randomized controlled trial.

The financial crisis to which our insurance systems are subject will probably force health authorities to no longer offer reimbursement for expensive devices if they have not been subjected to rigorous evaluation. While a waiting that, we can bet that we will still have the opportunity to read a new meta-analysis about flow diverters in the near future.
(D) E. Houdart Service de Neuroradiologie Hôpital Lariboisière Paris, France 